Karlsruher Institut für Technologie

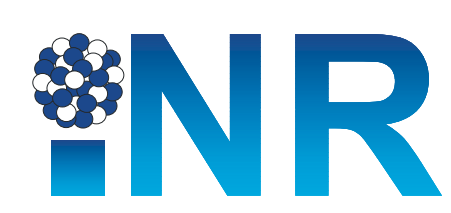

\title{
Propagation of Nuclear Data Uncertainties in PWR Pin-Cell Burnup Calculations via Stochastic Sampling
}

\section{Mercatali, Y. Alzaben, V. H. Sanchez Espinoza} Karlsruhe Institute of Technology

International Conference on Nuclear Engineering (ICONE26)

July 22-26, 2018 London (England)

Institute for Neutron Physics and Reactor Technology (INR)
Reactor Physics and Dynamic Group (RPD)

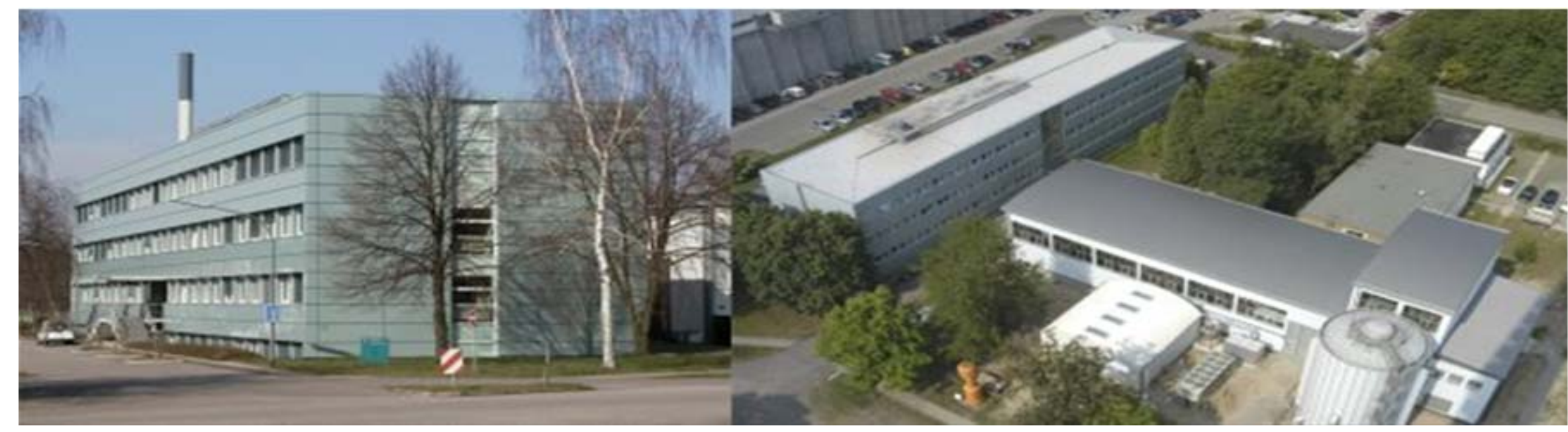




\section{Content}

- Introduction

- S/U analysis

- Methods

* The SAMPLER sequence

- Problem definition and modeling

* Calculation flowchart

Stochastic sampling vs. GPT methods

- Results

k-inf vs. irradiation time + uncertainties

Reaction rates

* Nuclide concentrations

- Summary 


\section{Preamble - "All models are wrong"}

- In computer modelling, errors and uncertainties inevitably arise due to the mathematical idealization of physical processes stemming from insufficient knowledge regarding accurate model forms as well as the precise value of input parameters

- Even the best models can only be as accurate as their input parameters

- Reality does not have parameters

- Before trusting the results obtained by simulations one has to make sure that they are representative of reality
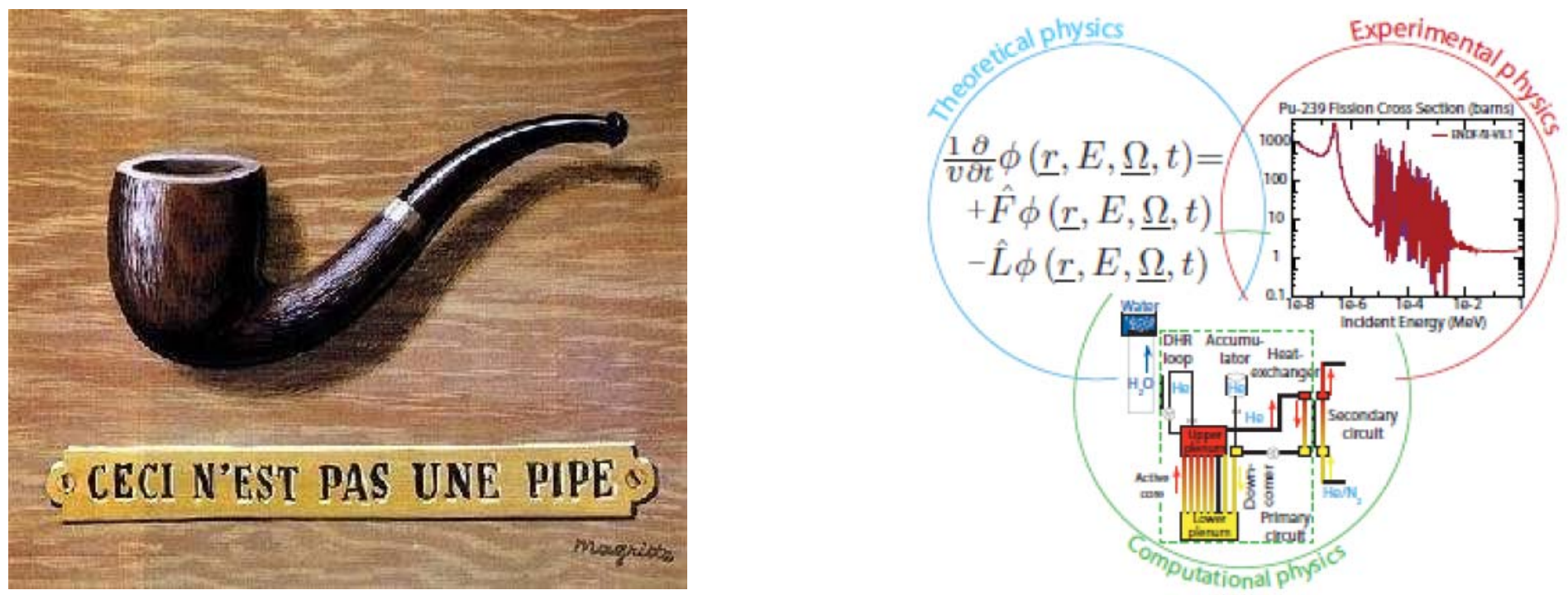


\section{Introduction}

- In recent year there has been an increasing demand from nuclear research, industry, safety, and regulatory bodies for best estimate predictions of LWRs performances to be provided with their confidence bound

- Understanding uncertainties of evaluated reactor parameters is important for introducing appropriate design margins and deciding where additional efforts should be undertaken to reduce those uncertainties

\section{OECD/UAM Benchmark for Uncertainty Analysis in Modeling for Design, Operation and Safety Analysis of LWRs}
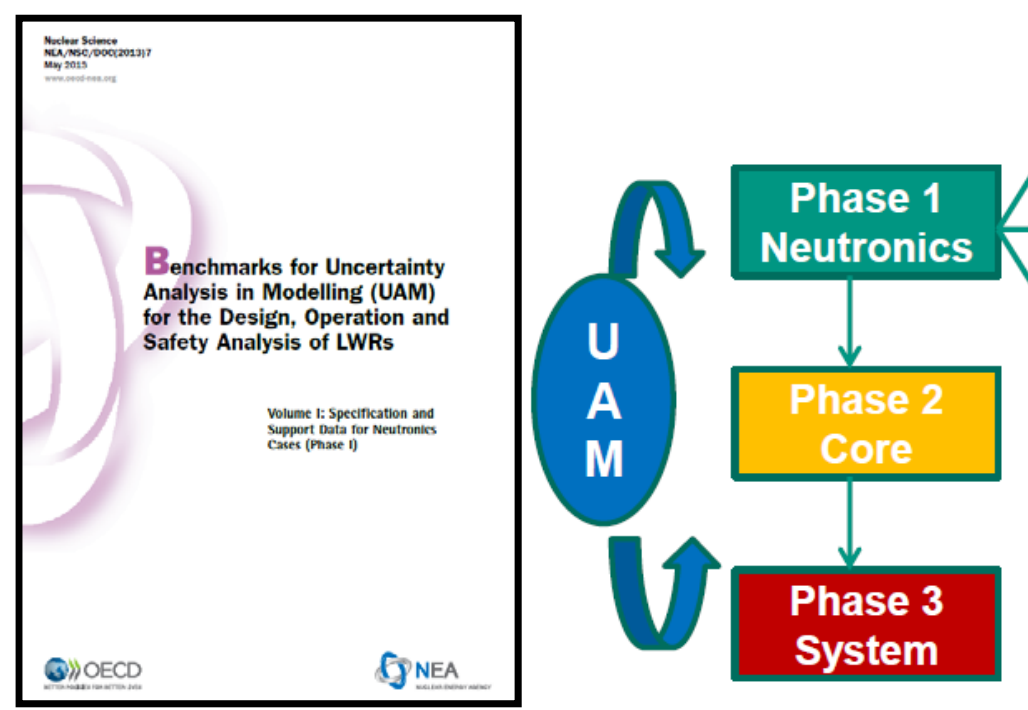

Exercises I-1

Cell Physics: derivation of the multi-group microscopic cross-section libraries and associated uncertainties

Lattice Physics: derivation of the few-group

macroscopic cross-section libraries and associated uncertainties

Exercises I-3

Core Physics: core steady state stand-alone neutronics calculations and associated uncertainties

Uncertainty propagation across multi-scale and multi-physics phenomena 


\section{S/U analysis}

- Different approaches

1. Direct perturbation

2. Perturbation/Generalized perturbation theories

3. Statistical sampling

4. Total Monte-Carlo (TMC)

- The statistical approach to uncertainty

* Uncertainty in input values described by PDF's

* The model output is a random variable whose distribution reflects the uncertainty in the output associated with the uncertainty in the input

* If one would know the probability distribution of the output one would be able to answer as precise as possible all questions about the likelihood of its values. The assumption of normal distribution is made

* Statistics offers the means to "quantify the goodness" of the output values

* Wilk's formula

$1-\alpha^{n} \geq \beta \quad$ (one sided)

$1-\alpha^{n}-n \cdot(1-\alpha) \cdot \alpha^{n-1} \geq \beta \quad($ two sided $)$

\begin{tabular}{|c|c|c|c|c|c|c|}
\hline & \multicolumn{3}{|c|}{$\begin{array}{c}\text { One-sided statistical } \\
\text { limits }\end{array}$} & \multicolumn{3}{c|}{$\begin{array}{c}\text { Two-sided statistical } \\
\text { limits }\end{array}$} \\
\hline $\boldsymbol{\beta} / \boldsymbol{\alpha}$ & $\mathbf{0 . 9 0}$ & $\mathbf{0 . 9 5}$ & $\mathbf{0 . 9 9}$ & $\mathbf{0 . 9 0}$ & $\mathbf{0 . 9 5}$ & $\mathbf{0 . 9 9}$ \\
\hline $\mathbf{0 . 9 0}$ & 22 & 45 & 230 & 38 & 77 & 388 \\
\hline $\mathbf{0 . 9 5}$ & 29 & 59 & 299 & 46 & 93 & 473 \\
\hline $\mathbf{0 . 9 9}$ & 44 & 90 & 459 & 64 & 130 & 662 \\
\hline
\end{tabular}




\section{The SAMPLER sequence}

- The SAMPLER module within SCALE provides uncertainty in any computed results from any SCALE sequence due to uncertainties in:

- Neutron cross sections

- Fission yield and decay data

- Geometry and composition

- SAMPLER employs sampling techniques to propagate $U Q$ for random uncertainties

* Given input PDF: $p(\bar{x})$

* Given QOls, forward model: $\bar{y}=\bar{F}(\bar{x})$

- Compute $\mathrm{N}$ realizations of $\bar{x}:\left\{\bar{x}_{1}, \bar{x}_{2}, \ldots . ., \bar{x}_{N}\right\}$

- Evaluate forward model of each realization $\left\{\bar{y}_{1}, \bar{y}_{2}, \ldots ., \bar{y}_{N}\right\}$

- Construct uncertainty quantities from sampledependent QOI data

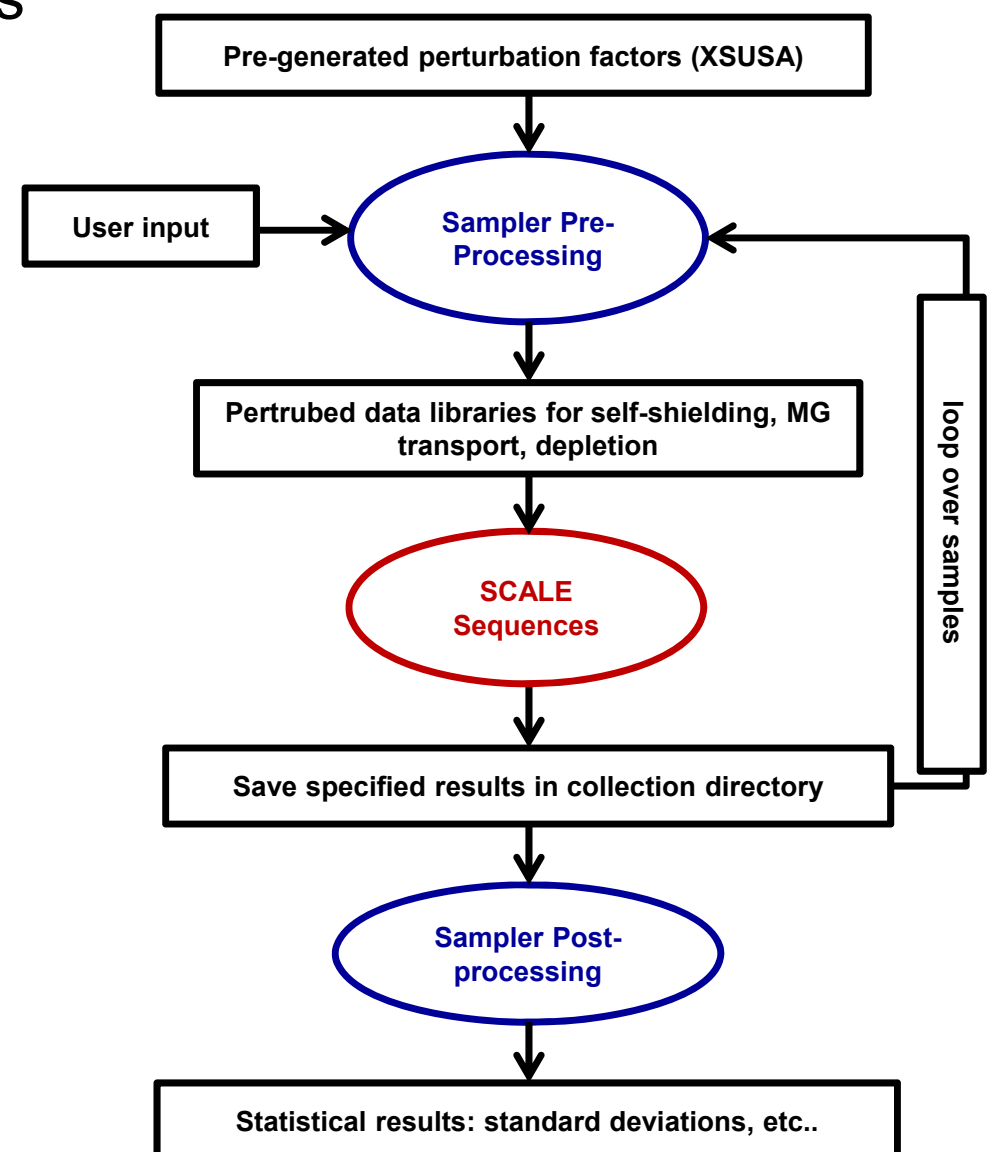

* Means, SDs, correlation coefficients, histograms 


\section{Problem definition and modeling}

- The UAM burn-up pin cell (Exercise l-1b)

- Power: $33.58 \mathrm{~kW} / \mathrm{kgU}$

- Final burn-up: 61.GWd/MTU

- Requested output

- K-inf

- One-group $(n, f)$ and $(n, y)$ reaction rates for $U$ and $P u$ isotopes

- Actinides and FPs isotopic concentrations

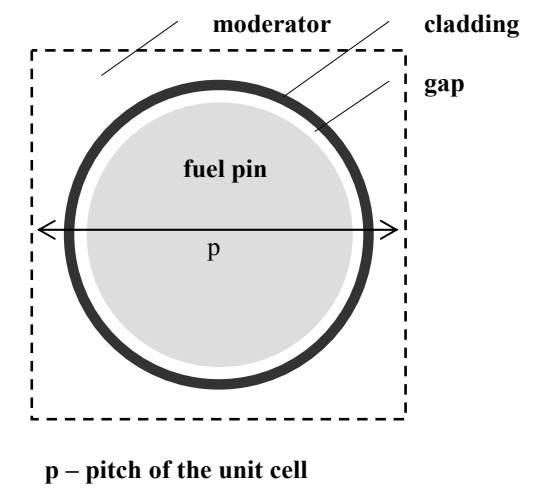

- The SCALE 6.2.2 code and ENDF/B.VII.1 nuclear data have been used

- TRITON sequence (NEWT + ORIGEN-S)

- NEWT used to calculate weighted burn-up dependent XS's

- BONAMI and CENTRM solvers for XS self-shielding

- 56-group ENDF/B.VII.1 XS library

- 56-group ENDF/B.VII.1 covariance library

- SAMPLER

- 1000 samples 


\section{Calculation flowchart}

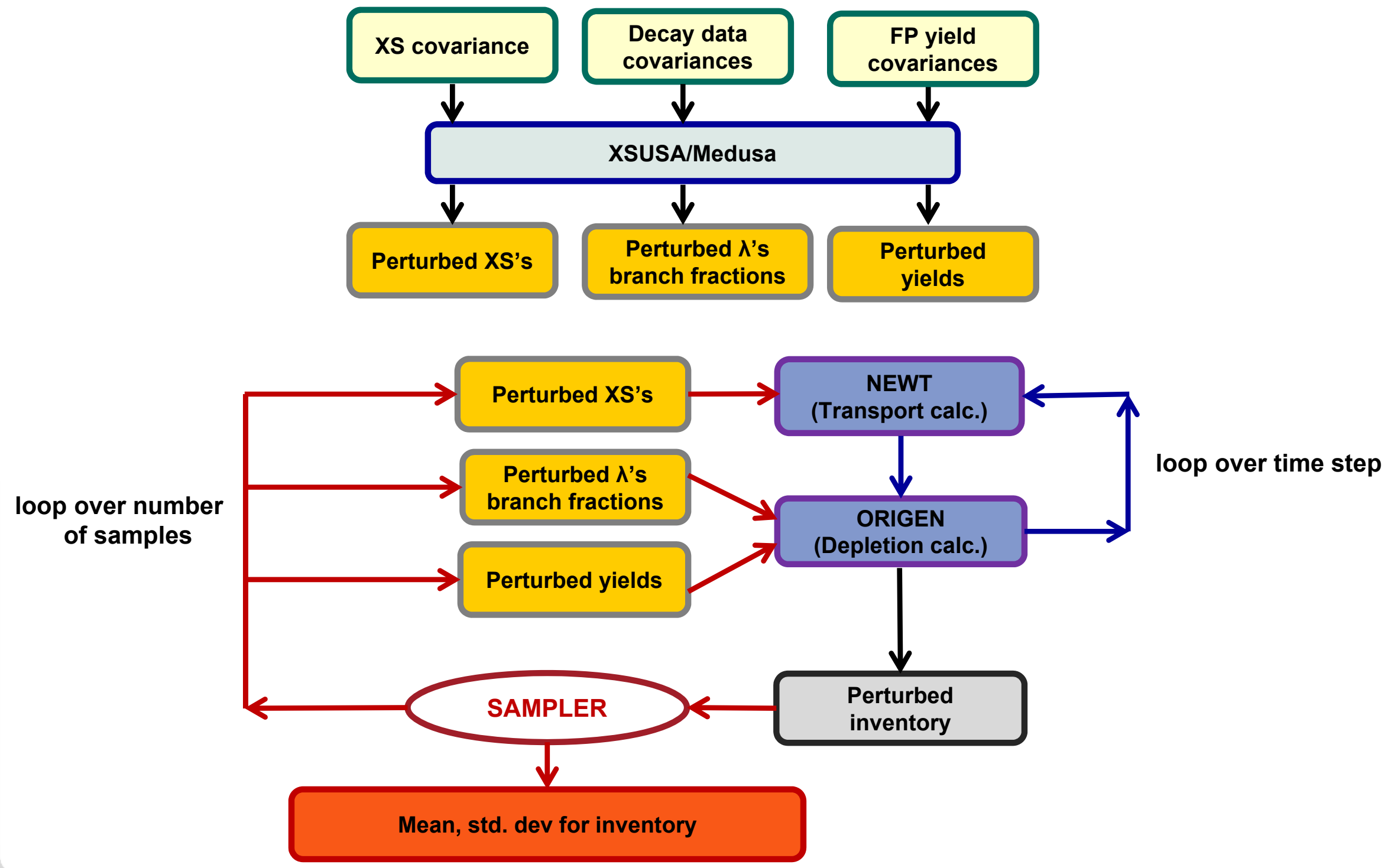




\section{Stochastic sampling vs. GPT}

- Comparison of the stochastic approach against the GPT approach showed an excellent agreement of the results $\left.{ }^{*}\right)$

\begin{tabular}{|c|c|c|c|c|c|}
\hline \multirow{3}{*}{$\begin{array}{l}\text { Test } \\
\text { case }\end{array}$} & \multirow{3}{*}{$\begin{array}{l}\text { Operating } \\
\text { condition }\end{array}$} & \multicolumn{4}{|c|}{ Calculation method } \\
\hline & & \multicolumn{2}{|c|}{ GPT } & \multicolumn{2}{|c|}{ Sampling } \\
\hline & & $k_{\text {inf }}$ & Uncert. & $\mathbf{k}_{\text {inf }}$ & Uncert. \\
\hline \multirow{2}{*}{ BWR } & HZP & 1.3428 & $6.8 \mathrm{E}-01$ & 1.3430 & $5.4 \mathrm{E}-01$ \\
\hline & HFP & 1.2249 & $5.9 \mathrm{E}-01$ & 1.2252 & $5.7 \mathrm{E}-01$ \\
\hline \multirow{2}{*}{ PWR } & HZP & 1.4253 & $5.4 \mathrm{E}-01$ & 1.4254 & $5.0 \mathrm{E}-01$ \\
\hline & HFP & 1.4063 & $5.5 \mathrm{E}-01$ & 1.4064 & $5.0 \mathrm{E}-01$ \\
\hline \multirow{2}{*}{ VVER } & HZP & 1.3457 & $5.8 \mathrm{E}-01$ & 1.3458 & $5.3 \mathrm{E}-01$ \\
\hline & HFP & 1.3276 & $5.8 \mathrm{E}-01$ & 1.3278 & $5.4 \mathrm{E}-01$ \\
\hline
\end{tabular}

\begin{tabular}{|c|c|c|c|c|}
\hline \multicolumn{5}{|c|}{ Test case: VVER - Kozloduy $\mathbf{6}$} \\
\hline \multirow{2}{*}{ Response } & \multicolumn{2}{|c|}{ TSUNAMI } & SAMPLER (N=93) \\
\cline { 2 - 5 } & Value & $\boldsymbol{\delta R / R}$ & Value & $\boldsymbol{\delta} / \mathbf{R}$ \\
\hline $\boldsymbol{\Sigma}_{\mathbf{f}}$ (gr. 1) & $2,411 \mathrm{E}-03$ & $5,071 \mathrm{E}-01$ & $2,435 \mathrm{E}-03$ & $5,505 \mathrm{E}-01$ \\
\hline $\boldsymbol{\Sigma}_{\mathbf{f}}$ (gr. 2) & $5,615 \mathrm{E}-02$ & $3,283 \mathrm{E}-01$ & $5,701 \mathrm{E}-02$ & $3,368 \mathrm{E}-01$ \\
\hline $\boldsymbol{\Sigma}_{\mathbf{a}}$ (gr. 1) & $1,408 \mathrm{E}-02$ & $1,343 \mathrm{E}+00$ & $1,410 \mathrm{E}-02$ & $9,076 \mathrm{E}-01$ \\
\hline $\boldsymbol{\Sigma}_{\mathbf{a}}$ (gr. 1) & $9,485 \mathrm{E}-02$ & $8,810 \mathrm{E}-01$ & $9,645 \mathrm{E}-02$ & $1,994 \mathrm{E}-01$ \\
\hline nu-fission (gr.1) & $6,159 \mathrm{E}-03$ & - & $6,218 \mathrm{E}-03$ & $8,418 \mathrm{E}-01$ \\
\hline nu-fission (gr.2) & $1,368 \mathrm{E}-01$ & - & $1,390 \mathrm{E}-01$ & $4,539 \mathrm{E}-01$ \\
\hline
\end{tabular}

\section{Pin-cells}

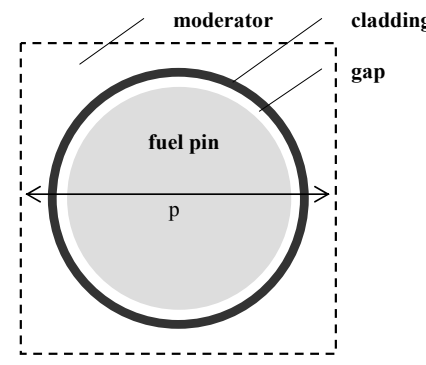

p - pitch of the unit cell

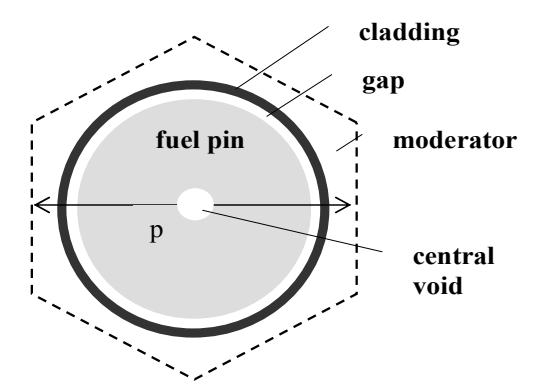

$p-$ pitch of the unit cell

\section{FAs}

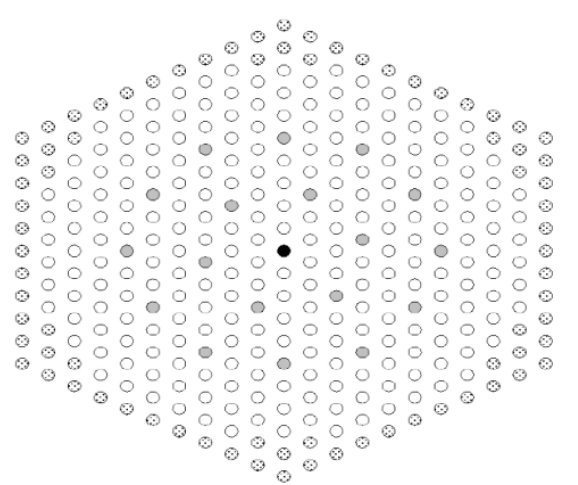

$\left(^{*}\right)$ L. Mercatali et al.: "SCALE Modeling of Selected Neutronics Test Problems within the OECD UAM LWR's Benchmark", Science and Technology of Nuclear Istallations, ID 573697, Volume 2013 (2013). 


\section{k-inf vs. irradiation time}

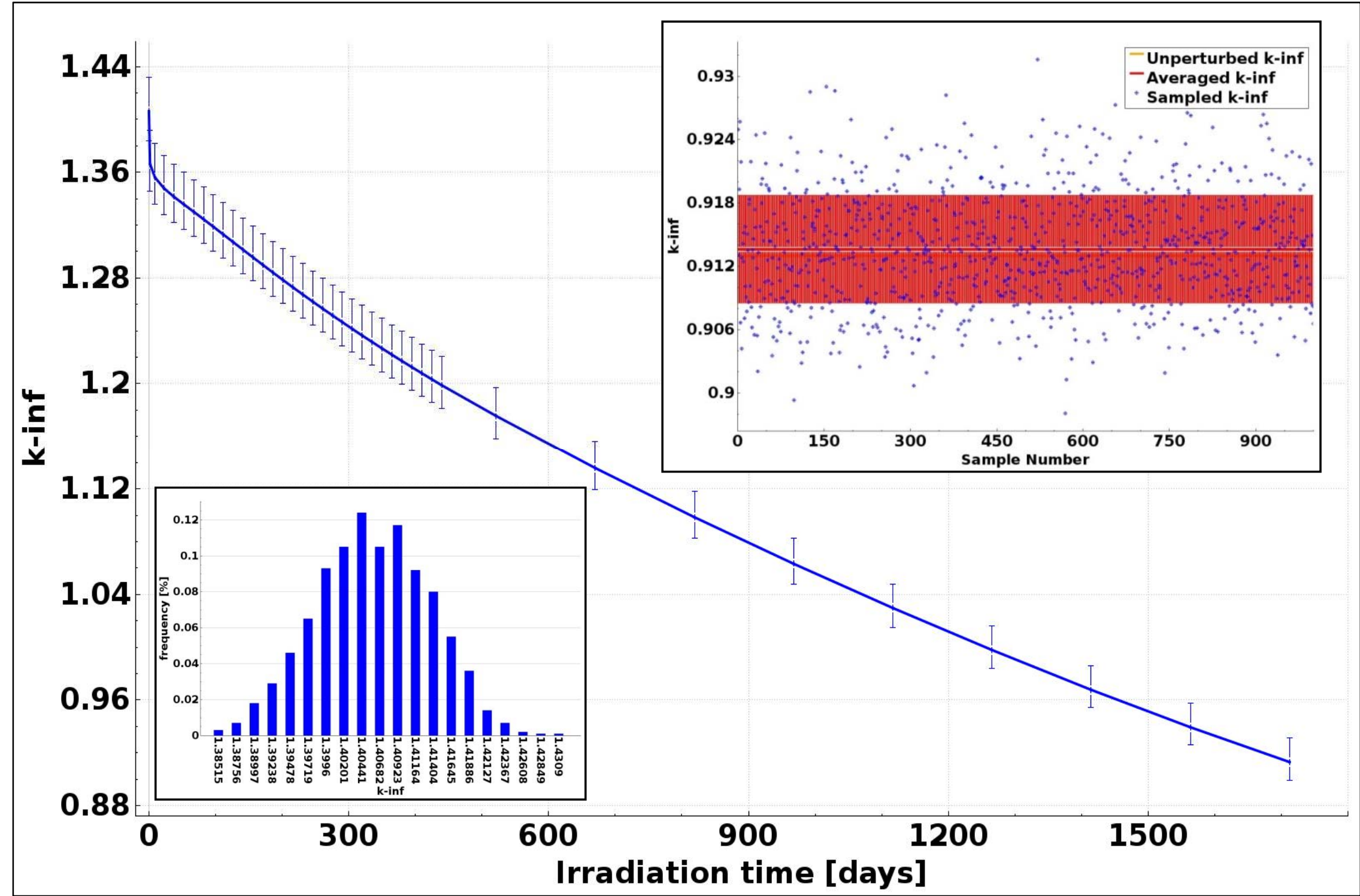

10 ICONE26, July 22-26, 2018 (London, UK) 


\section{Uncertainty on k-inf}

- Three sets of 1000 samples each:

1. XS perturbation

2. Decay data perturbation

3. FY perturbation

\begin{tabular}{c|c|c|c|}
$\begin{array}{c}\text { Burnup } \\
\text { [GWd/MTU] }\end{array}$ & $\begin{array}{c}\text { XS } \\
\text { RSD (pcm) }\end{array}$ & $\begin{array}{c}\text { FY } \\
\text { RSD (pcm) }\end{array}$ & $\begin{array}{c}\text { Decay } \\
\text { RSD (pcm) }\end{array}$ \\
\hline 0 & 545 & 0 & 0 \\
10 & 503 & 10 & 4 \\
20 & 495 & 13 & 3 \\
30 & 494 & 18 & 3 \\
40 & 505 & 23 & 3 \\
\hline 50 & 527 & 28 & 2 \\
60 & 560 & 31 & 2 \\
\hline
\end{tabular}

Contributions to the uncertainty on k-inf

- CPU time:19,46 days on a single processor 


\section{Concentrations of actinides}

- Uncertainty mainly due to XS

- Uncertainty increases with irradiation time
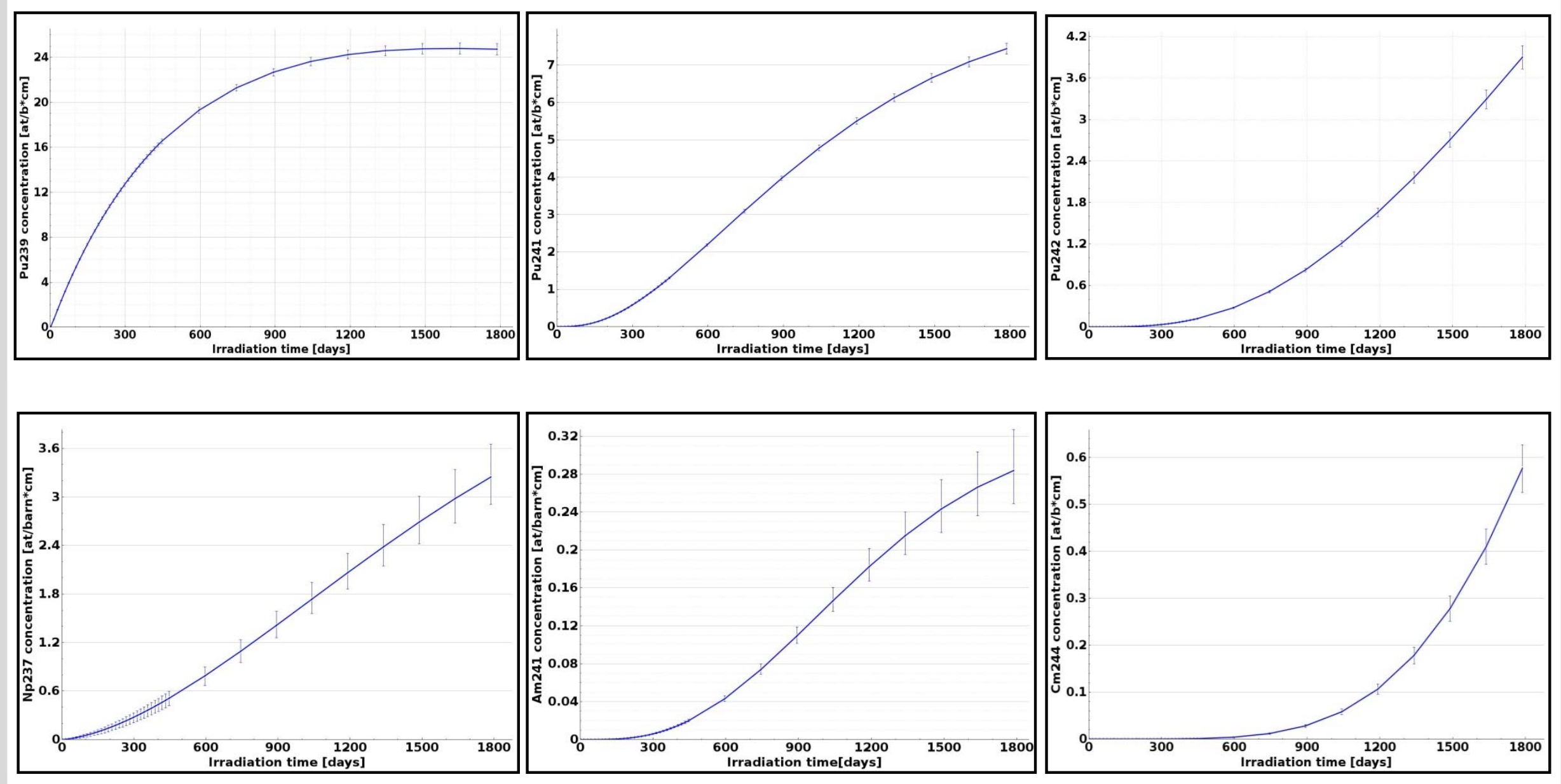

12 ICONE26, July 22-26, 2018 (London, UK) 


\section{Reaction rates}
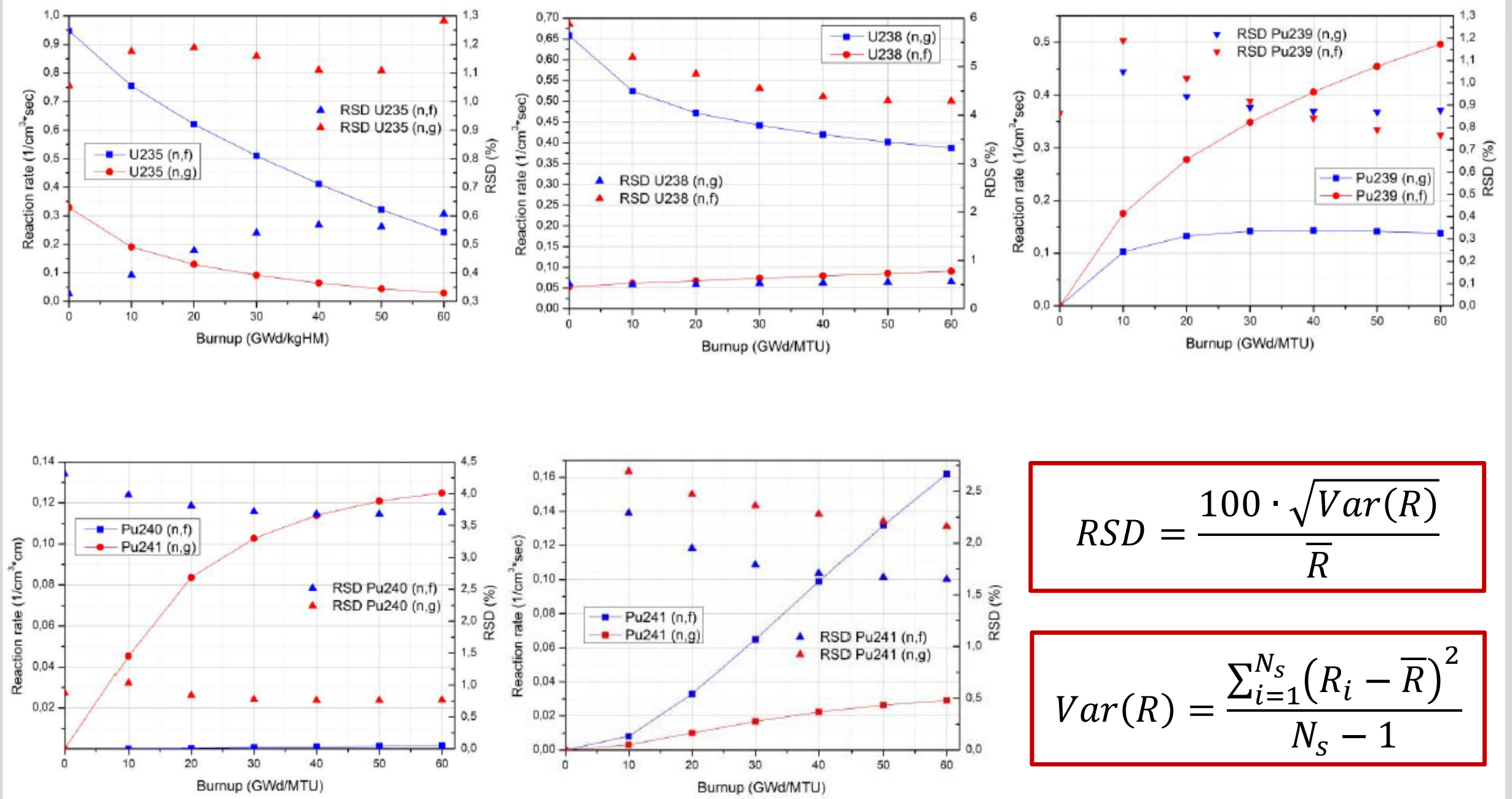

$$
\begin{gathered}
R S D=\frac{100 \cdot \sqrt{\operatorname{Var}(R)}}{\bar{R}} \\
\operatorname{Var}(R)=\frac{\sum_{i=1}^{N_{S}}\left(R_{i}-\bar{R}\right)^{2}}{N_{S}-1}
\end{gathered}
$$




\section{Uncertainties on nuclide concentrations}
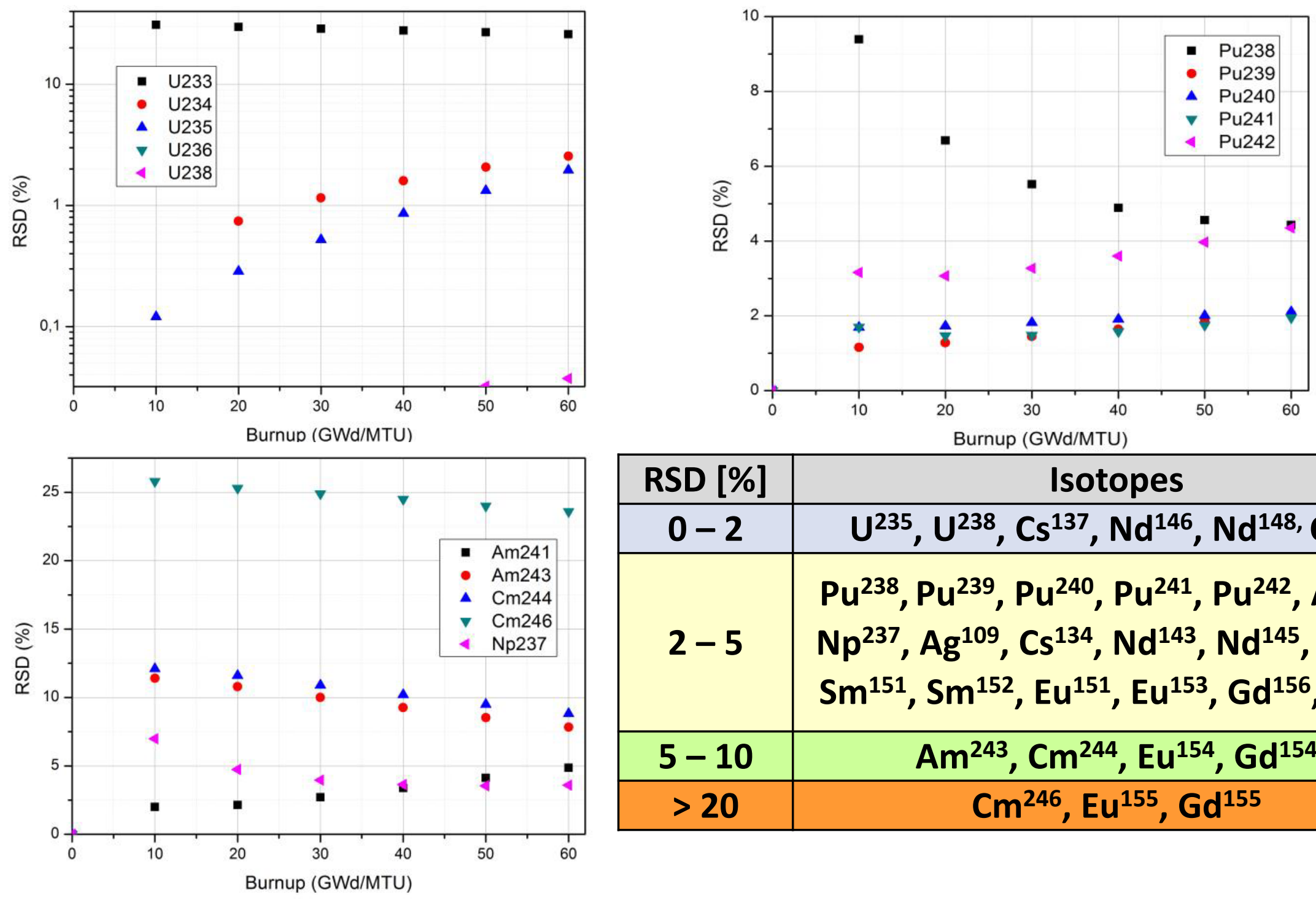

\begin{tabular}{|c|c|}
\hline RSD [\%] & Isotopes \\
\hline $0-2$ & $\mathrm{U}^{235}, \mathrm{U}^{238}, \mathrm{Cs}^{137}, \mathrm{Nd}^{146}, \mathrm{Nd}^{148}, \mathrm{Cs}^{137}$ \\
\hline & $\mathrm{Pu}^{238}, \mathrm{Pu}^{239}, \mathrm{Pu}^{240}, \mathrm{Pu}^{241}, \mathrm{Pu}^{242}, \mathrm{Am}^{241}$, \\
$2-5$ & $\mathrm{~Np}^{237}, \mathrm{Ag}^{109}, \mathrm{Cs}^{134}, \mathrm{Nd}^{143}, \mathrm{Nd}^{145}, \mathrm{Sm}^{148}$, \\
& $\mathrm{Sm}^{151}, \mathrm{Sm}^{152}, \mathrm{Eu}^{151}, \mathrm{Eu}^{153}, \mathrm{Gd}^{156}, \mathrm{Gd}^{158}$ \\
\hline $5-10$ & $\mathrm{Am}^{243}, \mathrm{Cm}^{244}, \mathrm{Eu}^{154}, \mathrm{Gd}^{154}$ \\
\hline$>20$ & $\mathrm{Cm}^{246}, \mathrm{Eu}^{155}, \mathrm{Gd}^{155}$ \\
\hline
\end{tabular}

14 ICONE26, July 22-26, 2018 (London, UK) 


\section{Correlation analysis}

- Pearson's coefficients $\left(P_{c}\right)$

- $\rho>0 \rightarrow$ correlation, $\rho<0 \rightarrow$ anti-correlation

- $|\rho|=1 \rightarrow$ perfect linear relationship

$$
P_{c}=\frac{\operatorname{Cov}(x, y)}{S D(x) \cdot S D(y)} \in[-1,1]
$$

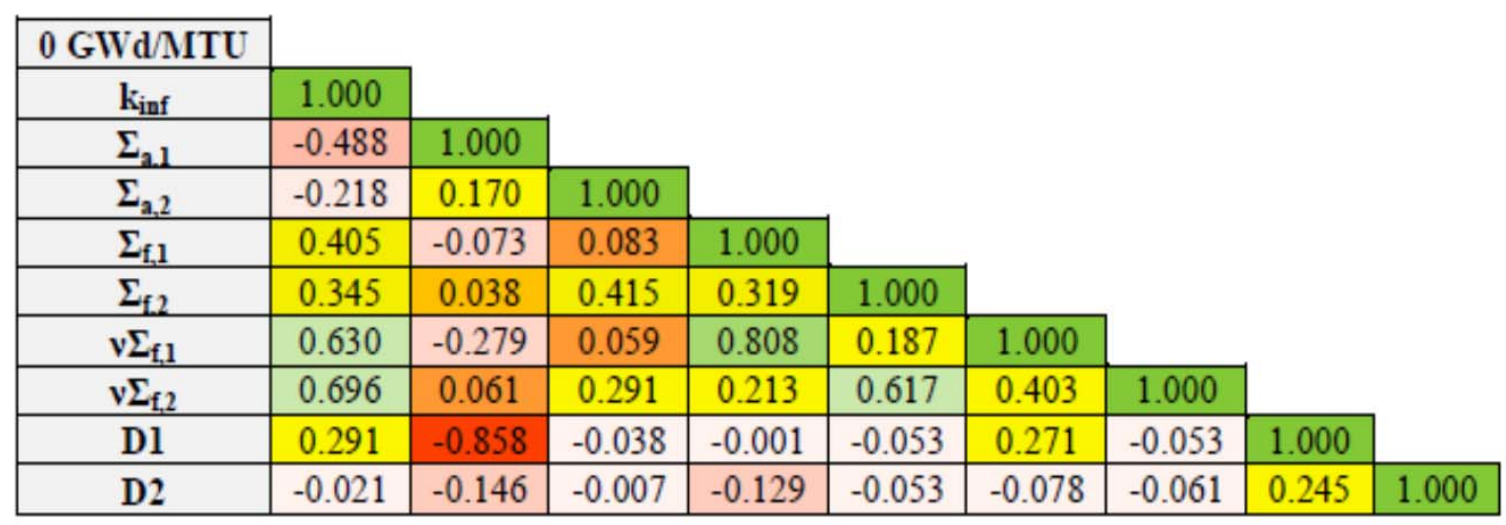

\begin{tabular}{|c|c|c|c|c|c|c|c|c|c|}
\hline $60 \mathrm{GWd} / \mathrm{MTU}$ & & & & & & & & & \\
\hline $\mathbf{k}_{\text {inf }}$ & 1.000 & & & & & & & & \\
\hline$\Sigma_{\text {a.1 }}$ & -0.600 & 1.000 & & & & & & & \\
\hline$\Sigma_{\mathrm{a} .2}$ & 0.051 & 0.401 & 1.000 & & & & & & \\
\hline$\Sigma_{\mathrm{f}, 1}$ & 0.539 & -0.262 & 0.678 & 1.000 & & & & & \\
\hline$\Sigma_{\mathrm{f} 2}$ & 0.280 & 0.348 & 0.942 & 0.682 & 1.000 & & & & \\
\hline$v \Sigma_{f, 1}$ & 0.558 & -0.265 & 0.638 & 0.972 & 0.636 & 1.000 & & & \\
\hline$v \Sigma_{\mathrm{f} 2}$ & 0.275 & 0.368 & 0.944 & 0.667 & 0.996 & 0.625 & 1.000 & & \\
\hline Dl & 0.610 & -0.779 & 0.127 & 0.678 & 0.163 & 0.658 & 0.143 & 1.000 & \\
\hline D2 & 0.087 & -0.291 & -0.267 & -0.151 & -0.239 & -0.143 & -0.244 & 0.227 & 1.000 \\
\hline
\end{tabular}

15 ICONE26, July 22-26, 2018 (London, UK) 


\section{Summary}

- A cell physics exercise has been performed, aiming to assess the uncertainties associated with the basic nuclear data in burn-up calculations for a typical PWR fuel pin-cell through a stochastic sampling approach

- Results obtained with the stochastic sampling method are in very good agreement with the ones obtained via GPT

- Uncertainties have been quantified as a function of the depletion time

- FY and decay constants have a negligible impact on the total uncertainty, the main contributor being the XS uncertainty

- The study represents the first step towards the uncertainty quantification for more complex burn-up problems (FAs, full core) 